\title{
Auditory-somatosensory multisensory interactions are spatially modulated by stimulated body surface and acoustic spectra
}

\author{
Ana Tajadura-Jiménez ${ }^{\mathrm{a}, \mathrm{b}, *}$, Norimichi Kitagawa ${ }^{\mathrm{b}}$, Aleksander Väljamäe ${ }^{\mathrm{a}, \mathrm{b}}$, Massimiliano Zampini $^{\mathrm{c}, \mathrm{d}}$, \\ Micah M. Murray ${ }^{\mathrm{e}, \mathrm{f}}$, Charles Spence ${ }^{\mathrm{g}}$ \\ a Division of Applied Acoustics, Chalmers University of Technology, Gothenburg, Sweden \\ ${ }^{\mathrm{b}}$ NTT Communication Science Laboratories, NTT Corporation, Japan \\ ${ }^{c}$ Department of Cognitive Sciences and Education, University of Trento, Rovereto (TN), Italy \\ d Center for Mind/Brain Sciences, University of Trento, Rovereto (TN), Italy \\ e Electroencephalography Brain Mapping Core, Center for Biomedical Imaging of Lausanne and Geneva, University of Lausanne, Switzerland \\ ${ }^{\mathrm{f}}$ Neuropsychology and Neurorehabilitation Service and Radiology Service, Centre Hospitalier Universitaire Vaudois and University of Lausanne, Switzerland \\ g Crossmodal Research Laboratory, Department of Experimental Psychology, Oxford University, UK
}

\section{A R T I C L E I N F O}

\section{Article history:}

Received 28 April 2008

Received in revised form 25 July 2008

Accepted 31 July 2008

Available online 6 August 2008

\section{Keywords:}

Multisensory

Crossmodal

Auditory

Somatosensory

Peripersonal space

Redundant Signals Effect (RSE)

\begin{abstract}
A B S T R A C T
Previous research has provided inconsistent results regarding the spatial modulation of auditorysomatosensory interactions. The present study reports three experiments designed to investigate the nature of these interactions in the space close to the head. Human participants made speeded detection responses to unimodal auditory, somatosensory, or simultaneous auditory-somatosensory stimuli. In Experiment 1, electrocutaneous stimuli were presented to either earlobe, while auditory stimuli were presented from the same versus opposite sides, and from one of two distances $(20 \mathrm{vs} .70 \mathrm{~cm})$ from the participant's head. The results demonstrated a spatial modulation of auditory-somatosensory interactions when auditory stimuli were presented from close to the head. In Experiment 2, electrocutaneous stimuli were delivered to the hands, which were placed either close to or far from the head, while the auditory stimuli were again presented at one of two distances. The results revealed that the spatial modulation observed in Experiment 1 was specific to the particular body part stimulated (head) rather than to the region of space (i.e. around the head) where the stimuli were presented. The results of Experiment 3 demonstrate that sounds that contain high-frequency components are particularly effective in eliciting this auditory-somatosensory spatial effect. Taken together, these findings help to resolve inconsistencies in the previous literature and suggest that auditory-somatosensory multisensory integration is modulated by the stimulated body surface and acoustic spectra of the stimuli presented.
\end{abstract}

(C) 2008 Elsevier Ltd. All rights reserved.

\section{Introduction}

People are often exposed to information occurring simultaneously in different sensory modalities, such as, for instance, when looking and listening to another person speaking. The brain frequently integrates these various sensory cues in order to give rise to unified multisensory perceptions (Ghazanfar \& Schroeder, 2006; Spence, 2007; Welch \& Warren, 1986). A great deal of the recent research has addressed the perceptual and neurophysiologic bases of multisensory processes and the impact of stimulus characteris-

\footnotetext{
* Corresponding author at: Chalmers University of Technology, Division of Applied Acoustics - Chalmers Room Acoustics Group, Sven Hultins gata 8a 41296, Gothenburg, Sweden. Tel.: +46 31772 2210; fax: +46 317722212.

E-mail address: ana.tajadura@ta.chalmers.se (A. Tajadura-Jiménez).
}

tics (e.g. Calvert, Spence, \& Stein, 2004; Driver \& Noesselt, 2008; Spence \& Driver, 2004).

External spatial proximity can have a dramatic impact on how information from different sensory modalities is integrated (at least for the 'spatial' modalities of vision, audition, and touch; e.g. Stein \& Meredith, 1993; Welch \& Warren, 1986). Electrophysiological recordings in animals have led researchers to formulate a "spatial rule" for multisensory integration (Stein \& Meredith, 1993) that stipulates that if stimuli in different sensory modalities are presented within the excitatory zones of a neuron's receptive field (RF) multisensory enhancement may well occur (i.e. provided that the other conditions for integration, such as temporal proximity, are also met). By contrast, if stimuli are not presented within the excitatory zones, then no multisensory enhancement or even response depression may be observed. According to this rule, what modulates multisensory integration is the neural RF organization rather 
than the external spatial position from which the stimuli themselves are presented; in other words, if the RFs are large enough, facilitatory interactions may even be expected to occur for stimuli that are spatially misaligned in external space (Murray et al., 2005; Wallace \& Stein, 2007; Zampini, Torresan, Spence, \& Murray, 2007).

Although it is still unclear whether there is a direct transposition of this rule based on neural encoding to behavioral indices of multisensory integration, psychophysical studies can nonetheless provide information concerning how spatial information regarding a stimulus might be represented and integrated. Inferences can then be drawn concerning the putative spatial representation at the level of a population of neurons. The present study addressed inconsistencies concerning the circumstances giving rise to spatially modulated interactions between auditory and somatosensory stimuli. Some research has failed to observe effects that are modulated by the external spatial alignment/proximity of stimuli, whereas others have demonstrated such effects.

Using a simple detection task, Murray et al. (2005) and Zampini et al. (2007) examined the redundant signals effect (RSE; i.e. the faster responding seen when pairs of multisensory stimuli, as compared to single unisensory stimuli, are presented) for auditory-somatosensory pairs. These pairs were either spatially aligned or were misaligned (either across left-right locations or front-back locations) and always involved somatosensory stimulation of the hands that were positioned away from the torso/head. Both studies observed significant RSEs for all stimulus combinations that exceeded predictions of probability summation. Moreover, there was no evidence of significant RT differences across different multisensory pairings (though see Lakatos, Chen, O'Connell, Mills, \& Schroeder, 2007, for evidence in monkeys suggesting there may be differences as a function of the stimulation of the ipsilateral versus the contralateral hand). In another study involving temporal order judgments (TOJs), no spatial modulation of performance was observed with somatosensory stimuli delivered to participants' fingers and auditory stimuli presented directly behind the fingers in front of participants (Zampini et al., 2005). In addition to these psychophysical findings, Murray et al. have provided electrical neuroimaging results documenting supraadditive neural response interactions at $50-90 \mathrm{~ms}$ post-stimulus onset that were localized to the caudal-medial (CM) auditory cortex in the hemisphere contralateral to the stimulated hand, irrespective of the location of the auditory stimulus. One proposition from these results is that the brain regions involved in processing auditory-somatosensory stimuli contain large spatial RFs, which allow for the integration of stimuli that happen to be separated by a relatively large distance in external space (Murray et al., 2005; Zampini et al., 2007).

By contrast, the spatial modulation of auditory-somatosensory TOJs and spatial discrimination of tactile targets in the presence of auditory distracters has been reported for auditory stimuli delivered in the region close to, and just behind, the head and somatosensory stimuli presented to participants' earlobes (Kitagawa, Zampini, \& Spence, 2005). In both varieties of task, the presentation of the multisensory stimuli in close spatial proximity impaired performance, whereas increasing the spatial separation reduced the interference effects. These results therefore suggest that the spatial modulation of auditory-somatosensory interactions might depend upon the particular region of space in which the stimuli are presented. That is, it may only occur in the space immediately next to and/or behind the head (see Kitagawa \& Spence, 2006, for a review).

One line of support for this proposition is the results of singlecell electrophysiological recordings from the intraparietal cortex of macaques (e.g. Graziano, Reiss, \& Gross, 1999; see also Graziano, Gross, Taylor, \& Moore, 2004, for a review), which have shown the existence of neurons with tactile RFs on the sides and back of the head that also respond to auditory stimuli presented from a position close to the head. Interestingly, in these studies, the distance from the listener to the auditory stimuli modulated neural indices of auditory-somatosensory interactions. The multisensory neurons no longer responded to the auditory stimulation when the sound was presented at a distance of $50 \mathrm{~cm}$ from the animal's head. Additional support is found in neuropsychological studies of patients suffering from tactile extinction (Farnè \& Làdavas, 2002; Làdavas \& Farnè, 2004) and auditory-tactile alloesthesia (Ortigue et al., 2005). For example, extinction patients have been shown to fail to detect contralesional tactile stimuli when ipsilesional auditory stimuli were simultaneously delivered, provided that the auditory stimuli were presented in the space extending only a few centimeters from the stimulated body surface. Likewise, recordings within the auditory cortex of the macaque monkey also provide indications that auditory-somatosensory interactions may be more prevalent when the head and/or neck is stimulated (Fu et al., 2003; see also Kayser, Petkov, Augath, \& Logothetis, 2005).

The present study examined the influence of the distance to auditory stimuli, the stimulated body surface and the nature of the auditory stimuli in the spatial modulation of auditory-somatosensory interactions as indexed by the RSE during a simple detection task in which the spatial information was task-irrelevant. To determine whether facilitative effects on behavior could be obtained with stimuli presented on the head and also to determine the impact of spatial alignment in this situation, Experiment 1 involved stimulation of the participants' earlobes with the auditory stimuli presented from two different distances from the participant (see Fig. 1A) and on either the same or opposite side of the body midline. To assess the importance of the stimulated body surface versus the stimulated region of space, Experiment 2

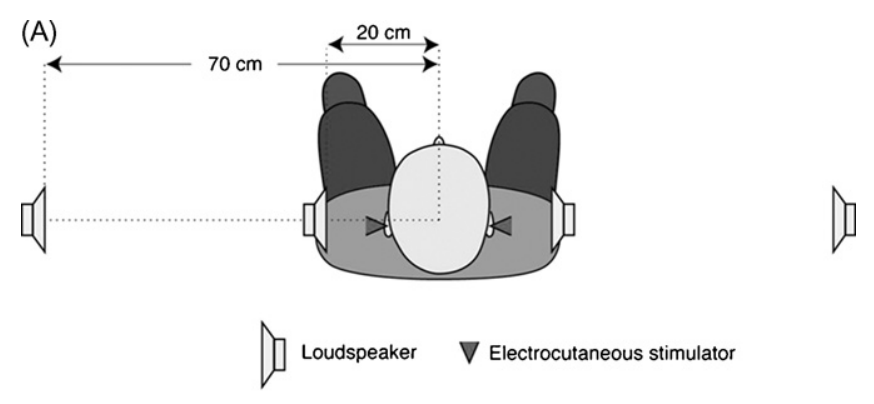

(B)

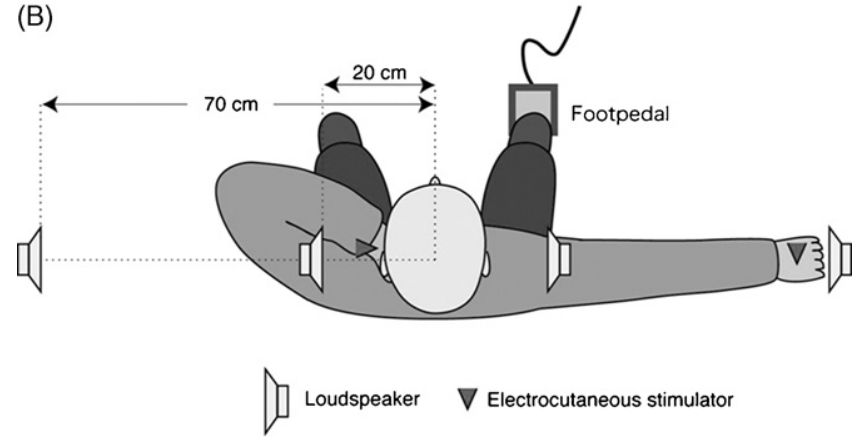

Fig. 1. Experimental setup used in Experiments 1 and 2 (bird's-eye view). (A) Experiment 1. Electrocutaneous stimulation was delivered to either left or right earlobe. Auditory stimulation was delivered from one of four loudspeaker cones. A response button held in the participant's hand was used for collecting participant's responses. (B) Experiment 2. Electrocutaneous stimulation was delivered to either hand, with the participant's arm placed in one of two possible postures (flexed vs. outstretched). The auditory stimulation was delivered from one of four loudspeaker cones. The participant's responses were registered by means of a footpedal. 
presented somatosensory stimuli to the hands of the participants who adopted a posture in which one of their hands was placed on the side of their neck (i.e. close to the head) and the other was outstretched to the side (i.e. far from the head; see Fig. 1B). The auditory stimuli were either close to or far from the somatosensory stimulus location and were either on the same or opposite side of the body midline, just as in Experiment 1. Finally, because previous research has demonstrated that the acoustic features of stimuli can also impact patterns of auditory-somatosensory interactions (Làdavas \& Farnè, 2004; Graziano et al., 1999; Kitagawa et al., 2005), Experiment 3 varied the frequency band-pass of the presented sounds.

\section{Methods}

All of the experiments reported here were conducted in accordance with the ethical standards laid down in the 1964 Declaration of Helsinki and approved by the ethics committee of the NTT Communication Science Laboratories. The participants were paid for their time and gave their informed consent prior to their inclusion in the study.

\subsection{Experiment 1}

\subsubsection{Participants}

Fifteen participants (mean age 27 years; age range from 20 to 34 years; seven females) took part in the experiment. All of the participants had normal hearing and twelve of them were naïve as to the purposes of the study (three of the authors, AT, $\mathrm{NK}$, and AV took part in the experiment).

\subsubsection{Apparatus and materials}

The experiment was conducted in a dark room with the participants seated in a chair with their head fixed on a chin rest. A green light-emitting diode (LED), positioned in front of the participants at eye level and $2 \mathrm{~m}$ away from them, served as the fixation point. The somatosensory stimulus consisted of an electrocutaneous stimulus presented to the lower tip of the participant's left and right earlobes through two circular electrodes (diameter $1 \mathrm{~cm}$, attached to a gel sheet). A custom-made electric stimulator was used to deliver the stimuli. Electrocutaneous stimulation was chosen because it is completely silent (cf. Kitagawa et al., 2005). The electrocutaneous stimulation was a single pulse lasting for $50 \mathrm{~ms}$ and was presented at a level that was clearly suprathreshold, but not painful. The intensity of the stimulation was approximately $2 \mathrm{mV}$, although it was carefully adjusted individually prior to the experiment by means of a preliminary simple detection task.

The auditory stimuli were delivered from one of four identical custom-made loudspeakers, with an $8 \mathrm{~cm}$ diameter cone, placed 20 and $70 \mathrm{~cm}$ to the left and right of the center of the participant's head (see Fig. 1A). The 'near' loudspeakers were placed $10 \mathrm{~cm}$ above the participant's ear, while the 'far' loudspeakers were placed $10 \mathrm{~cm}$ below their ear in order to avoid the possibility that the near loudspeakers would attenuate the sound emitted by the far loudspeakers. The auditory stimuli consisted of $50 \mathrm{~ms}$ white noise bursts $(50 \mathrm{~dB}(\mathrm{~A})$ as measured at the participant's ear position). A $10 \mathrm{~ms}$ onset/offset ramp was applied to the auditory stimuli to prevent clipping. A response button held in the participant's hand was used for the detection task. Presentation ${ }^{\circledR}$ software (Version 9.90) was used to control stimulus delivery and record responses.

\subsubsection{Design}

The experiment contained both unisensory and multisensory conditions (14 conditions in total). The unisensory conditions consisted of either auditory (A) stimuli, originating from one of the four possible locations (near or far, and left or right), or somatosensory (S) stimuli alone, with the electrocutaneous stimulation presented to the participant's left or right earlobe. These resulted in six possible unisensory conditions. The somatosensory trials were presented twice as often as the auditory trials in order to make sure that the participants received an equivalent number of stimuli in each modality overall and thus avoided a possible effect of expectancy in favor of the auditory conditions. In the multisensory conditions, auditory-somatosensory (AS) stimulus pairs were presented simultaneously in any one of the eight possible stimulus configurations (the auditory target originated from any one of the four positions, and the somatosensory target was presented from either the participant's left or right earlobe). These multisensory stimuli could either be spatially 'aligned' (i.e. both stimuli presented to the same side of the participant) or spatially 'misaligned' (e.g. the sound presented to one side and the somatosensory stimulus to the other side). This resulted in the following factorial design for the multisensory conditions: 2 sound distances $($ far $/$ near $) \times 2$ sides $($ left/right $) \times 2$ configurations (aligned/misaligned)
2.1.4. Procedure

At the start of the experimental session, the electrocutaneous stimulators were positioned and tested to adjust the intensity of the stimulation to a suprathreshold level, characterized as the level where participants detected more than $95 \%$ of the delivered electrocutaneous stimuli (the precise level varied across participants). The participants were then instructed to centrally fixate the LED during each block of trials and to perform a simple detection task in which they made speeded responses to any stimulus, irrespective of whether it was unisensory or multisensory. The speed of responding was emphasized to participants, but they were also instructed to refrain from making anticipatory responses. Responses were made by pressing a button which the participants held in their right hand. The participants completed two practice blocks with 16 trials each to familiarize themselves with the paradigm. The first practice block contained only somatosensory stimuli and served to confirm that the intensity of the electrocutaneous stimulation was appropriate. Next, the participants completed two blocks of 160 experimental trials, with each stimulus configuration presented 10 times within the block except for the unisensory somatosensory conditions in which the number of the trials was doubled. The different stimulus conditions were presented pseudo-randomly, with an inter-stimulus interval that varied randomly over the range 1500-3500 ms. Each block of trials lasted on average for $10 \mathrm{~min}$. During the experiment, detection accuracy and RTs for each trial were collected.

\subsection{Experiment 2}

\subsubsection{Participants}

Twelve participants (mean age 28 years; age range from 20 to 35 years; three females) took part in the experiment. All had normal hearing and eleven of them were naïve as to the purposes of the study (one of the authors, NK, took part in Experiment 2; one participant, aside from NK, took part in both Experiments 1 and 2).

\subsubsection{Apparatus and materials}

The experimental setup was identical to that used in Experiment 1, with the sole exception that the electrocutaneous stimuli were now delivered to the back of participants' hands, and that a footpedal was used for registering participants' responses.

\subsubsection{Design}

The experimental design resembled that used in Experiment 1 with both unisensory and multisensory conditions (28 different conditions in total). Unisensory conditions consisted of either auditory (A) stimuli, with the sound originating from one of the four possible locations (near or far, on the left or right), or somatosensory (S) stimuli (presented to the left or right hand) with two different arm postures (forearm flexed so that the hand held the side of the neck or outstretched to the far loudspeaker). This resulted in 12 possible unisensory conditions. The number of presentations of somatosensory trials was doubled in order to equal that of the auditory trials. The factorial design for the multisensory conditions was as follows: 2 arm postures (flexed/outstretched) $\times 2$ sound distances (near/far) $\times 2$ sides (left/right) $\times 2$ configurations (aligned/misaligned). Data from left and right sides were collapsed to rule out any effects of proprioceptive information that differed with arm posture and any sensitivity differences between the hands (cf. Zampini et al., 2007).

\subsubsection{Procedure}

The procedure was identical to that used in Experiment 1, except that the participants had to adopt a posture in which one of their arms was flexed, with the hand placed on the side of their neck (i.e. the position where the electrocutaneous stimuli were presented was just below the ear), and the other one outstretched to the far loudspeaker (the hand was placed just below the loudspeaker to avoid sound attenuation; see Fig. 1B). Which arm participants flexed or outstretched first was counterbalanced across the blocks of trials in order to ensure that effects followed from the spatial positions of the hands rather than any differences between somatosensory sensitivity of the participant's hands. The participants pressed the footpedal continuously throughout the trials and they released it as rapidly as possible whenever they detected a stimulus.

\subsection{Experiment 3}

\subsubsection{Participants}

Nine participants (mean age 24 years; age range from 20 to 34 years; three females) took part in the experiment. All had normal hearing and seven of them were naïve as to the purposes of the study (the two other participants were authors NK, AV).

\subsubsection{Apparatus and materials}

The experimental setup was identical to that used in Experiment 1, except that in this case only the 'near' loudspeakers $(20 \mathrm{~cm}$ to the left and right of the participant's midline) were used. A response button held in the hand was used for participants' response. 
The auditory stimuli, but not the electrocutaneous stimuli, differed from those used in Experiment 1. In this case, two types of auditory stimuli were used. 'Low frequency' sounds were band-pass filtered $(100-920 \mathrm{~Hz})$ noise bursts of $50 \mathrm{~ms}$ duration ( $40 \mathrm{~dB}(\mathrm{~A})$ as measured from participant's ear position). 'High frequency' sounds were band-pass filtered (13-17 kHz) noise burst of $50 \mathrm{~ms}$ duration $(50 \mathrm{~dB}(\mathrm{~A})$ as measured from participants' ear position). An onset/offset half-Hanning window ramp of $10 \mathrm{~ms}$ was applied to avoid clicks and clipping. Curves of equal loudness as a function of frequency as described by the International Organization for Standardization (ISO 226) were used to determine the appropriate sound pressure levels for both types of sounds to be perceived as being of the same loudness.

\subsubsection{Design}

The experimental design resembled that in Experiments 1 and 2 with both unisensory and multisensory conditions (14 different conditions in total). Unisensory conditions consisted of either auditory stimulus alone, with sound being 'high' or 'low' frequency and originating in one out of the two possible locations (left or right, at 'near' loudspeakers), or somatosensory stimulus alone (delivered to the left or right earlobe). These resulted in six possible unisensory conditions. The number of presentations of somatosensory trials was doubled in order to equal that of auditory trials. The factorial design for the multisensory conditions was the following: 2 sound frequencies (high/low) $\times 2$ sides (left/right) $\times 2$ configurations (aligned/misaligned).

\subsubsection{Procedure}

The procedure was identical to that of Experiment 1.

\subsection{Data analyses for all experiments}

RTs from each experiment were analyzed separately. Only RTs between 150 and 1000 ms were analyzed (cf. Murray et al., 2005; Zampini et al., 2007). Repeated measures analyses of variance (ANOVA) were performed on the RT data from the various different conditions. The within-participant factors differed for each of the experiments. Alpha level was fixed at 0.05 for all statistical tests. Greenhouse-Geisser correction was used to correct for unequal variances. Significant effects by ANOVA were followed by paired $t$-test comparisons. In addition, scatter plots contrasting the RTs for each participant for the two different multisensory configurations (aligned/misaligned) were produced, in order to visualize a possible systematic difference depending on alignment.

A further analysis was performed to test whether the improvement of RTs observed in the multisensory conditions, when compared to the unisensory conditions, could be explained in terms of neural response interactions. Presenting redundant signals simultaneously to various sensory channels often leads to a faster detection of an event than when a signal is presented to just a single channel. Under certain conditions, this RSE can be explained simply by probability summation. That is, the "separate-activation" or "race" models state (Raab, 1962) that when a signal is presented redundantly to two sensory channels, the faster of the two processes activates the response. On the other hand, there are cases in which the responses to redundant signals cannot be predicted by the race model because they are faster than what could have been achieved by processing the different channels of sensory information independently. "Co-activation" models (e.g. Lamarre, Busby, \& Spidalieri, 1983; Miller, 1982) attribute this effect to interactions taking place at a neural level contributing to the response.

We used Miller's inequality (Miller, 1982) to test whether the improvement of RTs in the multisensory conditions could be due simply to statistical facilitation (race model). The procedure for this analysis has been described elsewhere (Martuzzi et al., 2007; Murray, Foxe, Higgins, Javitt, \& Schroeder, 2001; Romei, Murray, Merabet, \& Thut, 2007). In brief, this procedure involved the following steps: calculate the probability distributions and normalize them in terms of the percentile of the range of RTs for each participant across all multisensory conditions (bin widths of $5 \%$ were used in the present study); compose a 20-point cumulative probability function for each condition formed by the average across participants; calculate the values predicted by the race model for each $5 \%$ bin in each multisensory condition (these modeled values equal the sum of the probabilities for each single-signal condition minus their joint probability); compare the actual probability of the multisensory conditions with their modeled values. In those cases where the probability predicted by the model is exceeded, it can be concluded that the race model cannot account for the facilitation in the redundant signals condition, thus supporting a neural coactivation model.

\section{Results}

\subsection{Experiment 1: effect of distance to sound on detection of auditory-somatosensory stimulus pairs}

On average, the participants detected $99 \pm 2.5 \%( \pm$ standard deviation (S.D.) indicated) of all auditory-only (A) stimuli, $99.8 \pm 0.9 \%$ of the somatosensory $(\mathrm{S})$ stimuli, and $97.1 \pm 6.5 \%$ of the multisensory

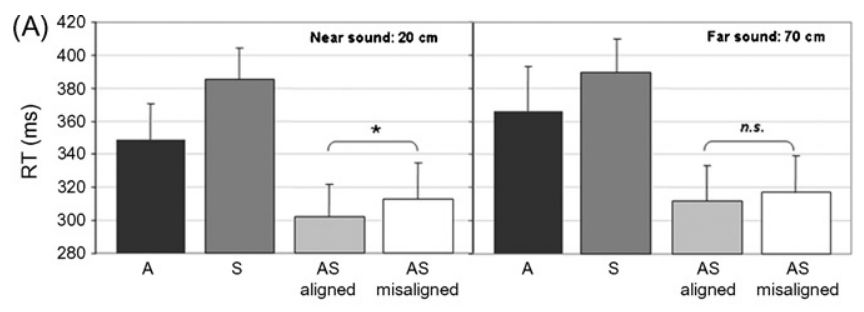

(B)

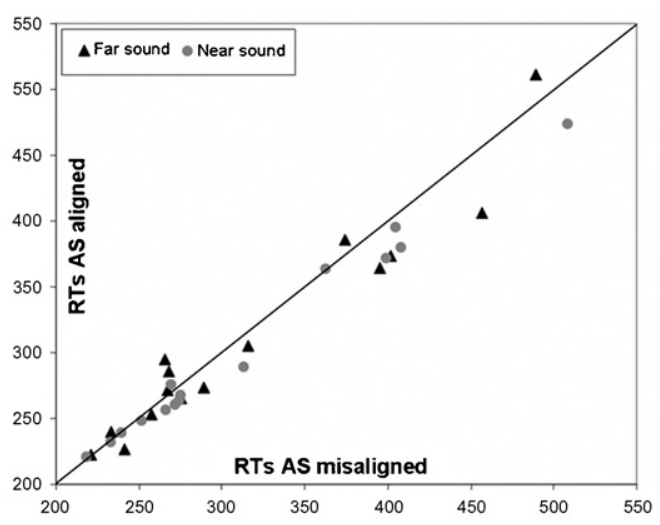

(C)

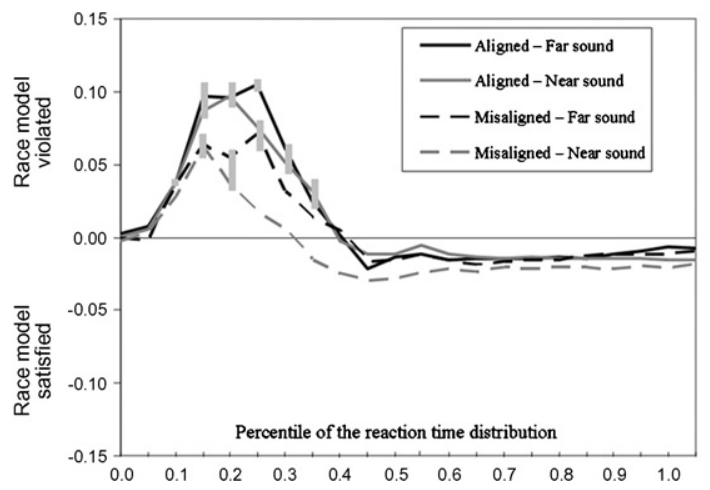

Fig. 2. Results of Experiment 1. (A) Mean RTs (in milliseconds) for the conditions in which the sound was presented from near (left panel) or far distance (right panel). The error bars indicate the standard errors of the mean. (B) Mean RTs (in milliseconds) for the AS aligned vs. AS misaligned conditions for each of the 15 participants. *marks a significant difference between conditions at $p<0.005$ level. (C) Results of applying Miller's (1982) inequality to the probability distributions of the RTs to the multisensory conditions. Whenever the probability predicted by the race model is exceeded (positive values in the graph), the race model cannot account for the facilitation in the redundant signals conditions, thus supporting a neural co-activation model. The grey rectangles indicate the percentiles of the probability distribution where the race model was significantly violated $(p<0.05)$ for each condition, as shown by additional analysis by paired $t$-test (one-tailed) comparisons.

(AS) stimulus pairs. A total of $97.5 \pm 3.1 \%$ of the trials from each participant were included in the data analyses (range: 89.1-100\%). A preliminary analysis was performed in order to examine whether the side of stimulation had a significant effect on participants' RTs. The results did not reach significance $(p>0.3)$, thus conditions that only differed in the side of presentation (left or right) were collapsed and further analyzed together.

The analysis of the results contained three separate ANOVAs examining the RTs. The first two ANOVAs tested, respectively, for a RSE in those conditions where the sound was presented from 'near' and 'far' from the participant. The within-participant factor for these ANOVAs was stimulus condition: A, S, AS aligned, or AS misaligned. The results (see Fig. 2A) showed that there was a significant main effect of stimulus condition $\left(F_{(2,24)}=69.3 ; p<0.001\right.$, for 'near', and $F_{(2,22)}=25.72 ; p<0.001$, for 'far' sound conditions). Follow-up planned comparisons (paired $t$-test) revealed that RTs 
Table 1

Results of follow-up planned comparisons between mean RTs for AS stimulus pairs for the different conditions over the three experiments

\begin{tabular}{|c|c|c|c|c|c|c|}
\hline & Somatosensory condition & Auditory condition & RSE? & AS aligned (ms) & AS misaligned (ms) & $t$-Value $(\mathrm{df}) ; p$-value \\
\hline Experiment 1 & Earlobe & $\begin{array}{l}\text { Near sound }(20 \mathrm{~cm}) \\
\text { Far sound }(70 \mathrm{~cm})\end{array}$ & $\begin{array}{l}\sqrt{ } \\
\sqrt{ }\end{array}$ & $\begin{array}{l}302 \\
312\end{array}$ & $\begin{array}{l}313 \\
317\end{array}$ & $\begin{array}{l}t_{14}=3.4 ; p=0.004^{* * *} \\
t_{14}=0.9 ; p=0.4 \text { (n.s.) }\end{array}$ \\
\hline Experiment 2 & $\begin{array}{l}\text { Hand - arm flexed } \\
\text { Hand - arm outstretched }\end{array}$ & $\begin{array}{l}\text { Near sound }(20 \mathrm{~cm}) \\
\text { Far sound }(70 \mathrm{~cm}) \\
\text { Near sound }(20 \mathrm{~cm}) \\
\text { Far sound }(70 \mathrm{~cm})\end{array}$ & $\begin{array}{l}\sqrt{ } \\
\sqrt{ } \\
\sqrt{ } \\
\sqrt{ }\end{array}$ & $\begin{array}{l}226 \\
224 \\
227 \\
224\end{array}$ & $\begin{array}{l}223 \\
225 \\
226 \\
225\end{array}$ & $\begin{array}{l}t_{11}=0.93 ; p=0.4 \text { (n.s.) } \\
t_{11}=0.3 ; p=0.77 \text { (n.s.) } \\
t_{11}=0.14 ; p=0.9 \text { (n.s.) } \\
t_{11}=0.36 ; p=0.73 \text { (n.s.) }\end{array}$ \\
\hline Experiment 3 & Earlobe & $\begin{array}{l}\text { High frequency }(20 \mathrm{~cm}) \\
\text { Low frequency }(20 \mathrm{~cm})\end{array}$ & $\begin{array}{l}\sqrt{ } \\
\sqrt{ }\end{array}$ & $\begin{array}{l}301 \\
299\end{array}$ & $\begin{array}{l}316 \\
310\end{array}$ & $\begin{array}{l}t_{8}=5.3 ; p=0.001^{* * *} \\
t_{8}=2.2 ; p=0.06 \text { (n.s.) }\end{array}$ \\
\hline
\end{tabular}

were significantly faster for multisensory conditions than for either unisensory condition (that is, a significant RSE was observed; $p<0.001)$. When sound was presented from 'near' distance, the participants responded more rapidly $\left(t_{14}=3.8 ; p<0.005\right)$ to the auditory than to the electrocutaneous unisensory targets. Moreover, in the 'near' sound conditions, RTs were facilitated for spatially aligned versus misaligned AS pairs $\left(t_{14}=3.4 ; p<0.005\right)$; however, this facilitation of performance was not observed in the 'far' sound conditions (see Table 1 for a summary of the comparisons for AS stimulus pairs for the three experiments).

A third ANOVA was conducted on the RTs from just the multisensory conditions using the within-participant factors of spatial alignment (aligned vs. misaligned) and distance between the location of the auditory stimuli and the ear (near vs. far). RTs were faster when the auditory and somatosensory stimuli were spatially aligned $\left(F_{(1,14)}=5.6 ; p=0.032\right)$. The effect of the distance of the auditory stimuli from the head was marginally significant $\left(F_{(1,14)}=4.3 ; p=0.057\right)$ with faster responses being reported when the auditory stimuli were presented from closer to the participants' heads (see Fig. 2B for a comparison of the mean RTs to aligned and misaligned conditions for each of the 15 participants). The interaction between the spatial alignment and distance was, however, not significant $\left(F_{(1,14)}=0.98\right.$; $p=0.34$ ).

In addition, we tested whether the RSE exceeded the statistical facilitation predicted by probability summation using Miller's inequality (Miller, 1982). In all of the multisensory conditions, paired $t$-test (one-tailed) comparisons between the actual and the modeled values revealed that the race model was violated at some point of the RT distribution (see Fig. 2C), thus supporting a neural co-activation model, as revealed. An ANOVA with withinparticipant factors of spatial alignment (aligned vs. misaligned), distance (near vs. far), and percentile of the RT distributions revealed that the violation was significantly larger $\left(F_{(1,14)}=7.35\right.$; $p=0.017$ ) for the spatially 'aligned' than for the spatially 'misaligned' conditions.

\subsection{Experiment 2: effect of stimulated body surface on detection of auditory-somatosensory stimulus pairs}

On average, the participants detected $99 \pm 2.8 \%$ ( \pm S.D. indicated $)$ of all auditory-only (A) stimuli, $92.9 \pm 11.5 \%$ of the somatosensory (S) stimuli, and $98.4 \pm 4.5 \%$ of the multisensory (AS) stimulus pairs. A total of $97.2 \pm 2.9 \%$ of the trials from each participant were included in the data analyses (range: $91.6-100 \%$ ). A preliminary analyses of the RT data from the unisensory conditions revealed a trend toward faster responses for the 'flexed' arm position and for the 'near' sound conditions although these effects did not reach significance ( $p=0.058$ for both cases).

When considering both unisensory and multisensory conditions, the analysis of the results consisted of four separate ANOVAs which examined the RTs in the different combinations of 'near' vs. 'far' sound conditions and 'flexed' vs. 'outstretched' arm posture. The within-participant factor was stimulus condition (A, S, AS aligned, AS misaligned). The four analyses revealed very similar results for all conditions (see Fig. 3A). That is, a significant effect of stimulus condition (for all analyses $p<0.001$ ). Follow-up comparisons revealed that participants responded significantly more rapidly $(p<0.001)$ to auditory than to somatosensory stimulation, and to multisensory stimuli than to either unisensory condition, irrespective of whether the multisensory stimuli were aligned or not. The general difference in RTs for this and Experiment 1 (compare Figs. 2A and $3 \mathrm{~A}$ ) might be accounted for by differences in neural conduction latencies for stimuli presented to the earlobe versus hand, as well as to differences in how the motor response was executed (e.g. see Bergenheim, Johansson, Granlund, \& Pedersen, 1996).

A further ANOVA was conducted on just the RTs for multisensory stimulus pairs. The within-participant factors in this analysis were stimulus condition (AS aligned, AS misaligned), sound distance (near, far) and arm posture (flexed, outstretched). This analysis revealed no significant effects or interactions (see Fig. 3B for a comparison of the mean RTs to aligned and misaligned conditions for each of the 12 participants).

In addition, we used Miller's (1982) inequality to test whether the RSE exceeded the statistical facilitation. Results from the comparison between the actual and the modeled values are presented in Fig. $3 \mathrm{C}$ for both arm positions ('flexed', in the left panel, and 'outstretched', in the right panel). Paired $t$-test (one-tailed) comparisons between the actual and the modeled values revealed that the violation of the model was significant $(p<0.05)$ in all conditions at some points in the RT distribution, thus supporting a neural co-activation model. An ANOVA with within-participant factors of arm posture (flexed vs. outstretched), spatial alignment (aligned vs. misaligned), sound distance (near vs. far), and percentile of the RT distributions revealed that there was no statistical difference in the violation of the race model between conditions.

\subsection{Experiment 3: effect of auditory frequency band on detection of auditory-somatosensory stimulus pairs}

On average, the participants detected $99.6 \pm 1.4 \%( \pm$ S.D. indicated) of all auditory-only (A) stimuli (no significant differences in percentage of detection between high and low frequency sounds), $96.8 \pm 8.5 \%$ of the somatosensory (S) stimuli, and $99.4 \pm 1.5 \%$ of the multisensory (AS) stimulus pairs. A total of $98.4 \pm 2 \%$ of the trials from each participant were included in the analyses (range: 93.1-100\%). A preliminary analysis was performed in order to examine whether the side of stimulation had a significant effect on the participants' RTs. The results did not reach significance, thus the data from the conditions that only differed in the side of presentation (left or right) were collapsed.

The analysis of the results entailed three separate ANOVAs which examined the RTs for the different conditions. The first 


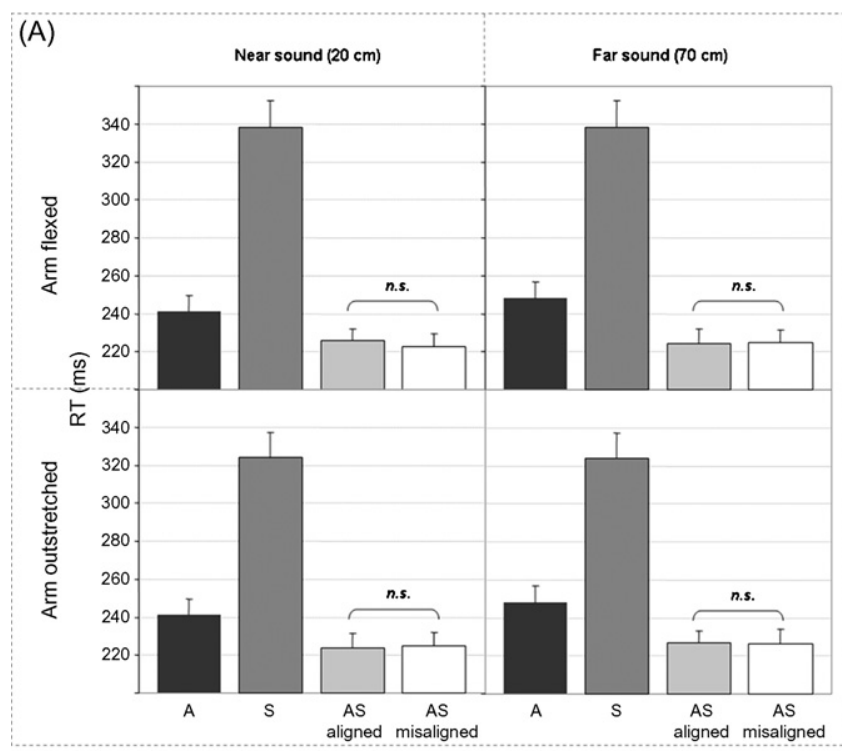

(B)
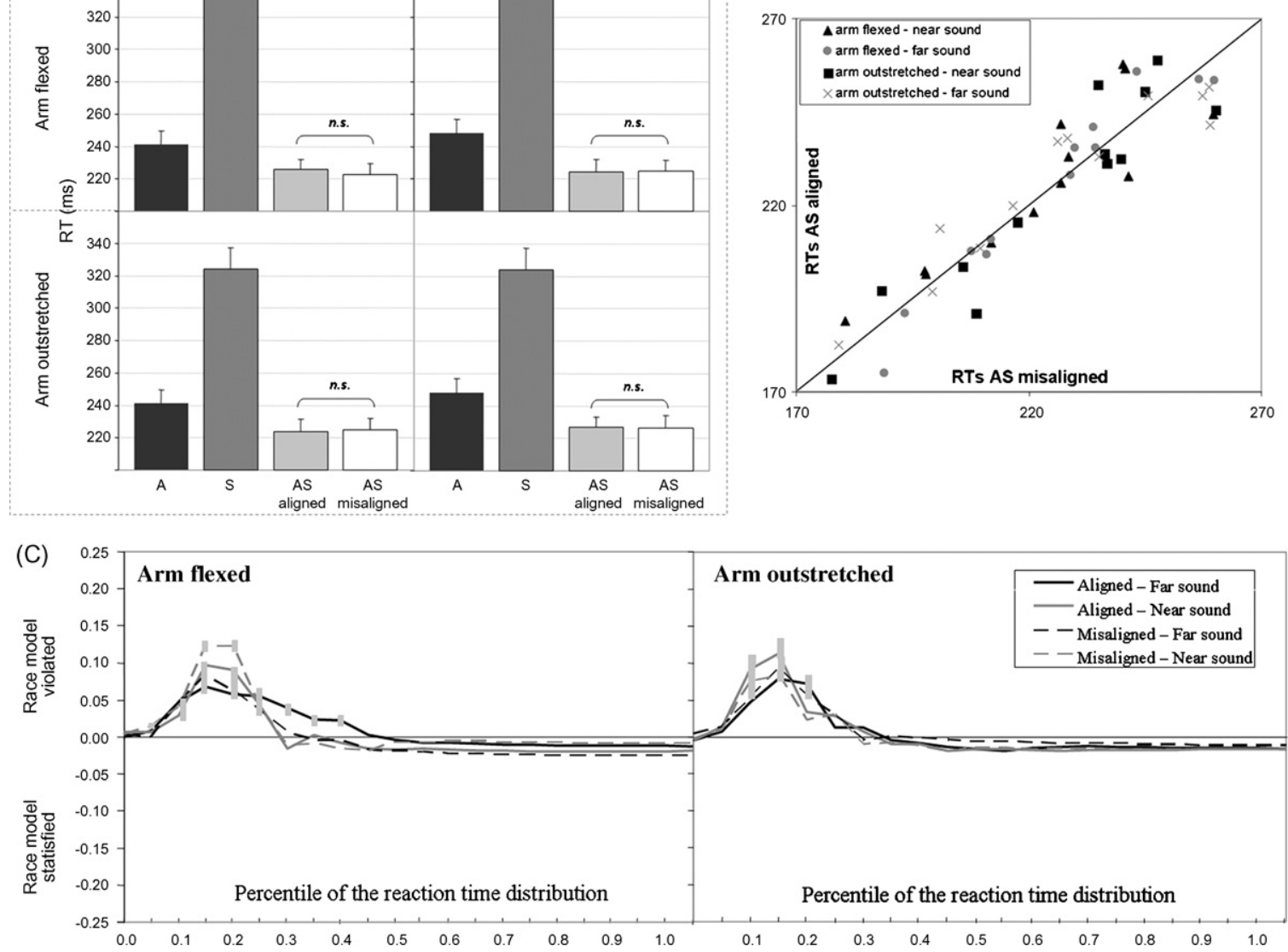

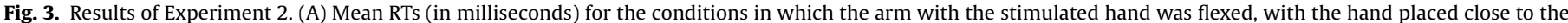

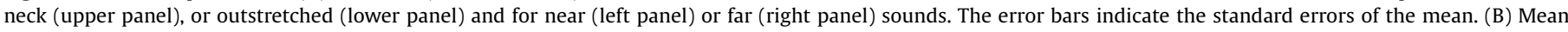

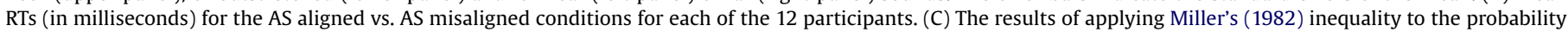
distributions of the RTs to the multisensory conditions.

two ANOVAs tested, respectively, for a RSE in the 'low' and 'high' frequency conditions. The within-participants factor was stimulus condition (A, S, AS aligned, AS misaligned). The results (see Fig. $4 \mathrm{~A}$ and Table 1 ) revealed that there was a significant effect of stimulus condition $\left(F_{(1,10)}=30.4 ; p<0.001\right.$, for 'low frequency', and $F_{(1,8)}=40.6 ; p<0.001$, for 'high frequency' sound conditions). Follow-up comparisons revealed that there was no significant difference between the auditory and somatosensory unisensory conditions, and that the participants responded significantly more rapidly to multisensory stimuli than to either unisensory condition $(p<0.001)$. Presenting the multisensory stimuli aligned as compared to misaligned in the multisensory conditions yielded faster responses for the 'high frequency' conditions $\left(t_{8}=5.4 ; p<0.001\right)$; however, no significant facilitation $(p>0.5)$ was observed in the 'low frequency' conditions.

A third ANOVA was conducted in order to examine whether the facilitation attributable to the RSE was larger when both modalities of stimulation were presented aligned, and whether the effect differed between the 'high frequency' and 'low frequency' sound conditions. The within-participant factors were multisensory stimulus condition (AS aligned, AS misaligned) and auditory frequency (high vs. low). The results showed that participants responded significantly more rapidly when the auditory and somatosensory stimuli were aligned than when they were misaligned $\left(F_{(1,7)}=25.6\right.$; $p<0.001)$, but neither the frequency factor $(p>0.26)$, nor the interaction between the two factors, multisensory stimulus condition and frequency, reached statistical significance (see Fig. 4B for a comparison of the mean RTs to aligned and misaligned conditions for each of the 9 participants). Note that although in the auditory-alone conditions, RTs for the 'high frequency' sounds were slower than for the 'low frequency' sounds (see Fig. 4A), this did not result in a significant difference between the multisensory conditions in which high versus low frequency sounds were presented. That is, a larger multisensory gain was observed in the high frequency condition (a point to which we return in Section 4).

In this experiment, paired $t$-test (one-tailed) comparisons between the actual and the modeled values revealed that the race model was violated in all conditions at some points in the RT distribution, thus supporting a neural co-activation model (see Fig. 4C). An ANOVA with within-participant factors of spatial alignment (aligned vs. misaligned), auditory frequency (high vs. low), and percentile of the RT distributions revealed that the violation was significantly larger $\left(F_{(1,8)}=7.43 ; p=0.026\right)$ for the spatially 'aligned' than for the spatially 'misaligned' conditions, and for the 'high frequency' compared to the 'low frequency' conditions $\left(F_{(1,8)}=6.5\right.$; $p=0.034)$.

\section{Discussion}

The results of the present study suggest that spatial modulation of auditory-somatosensory interactions, with integration 


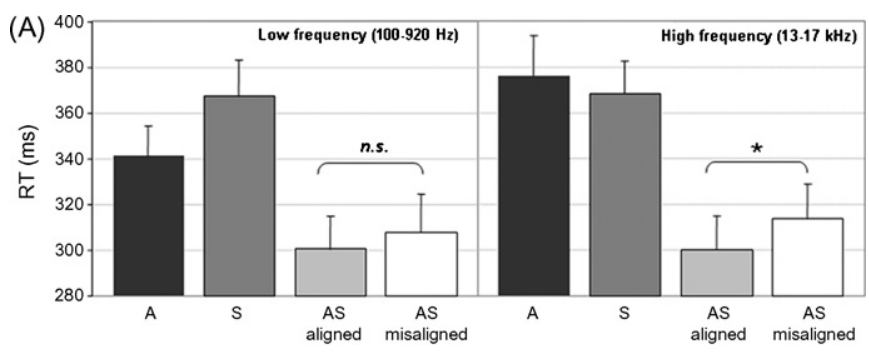

(B)

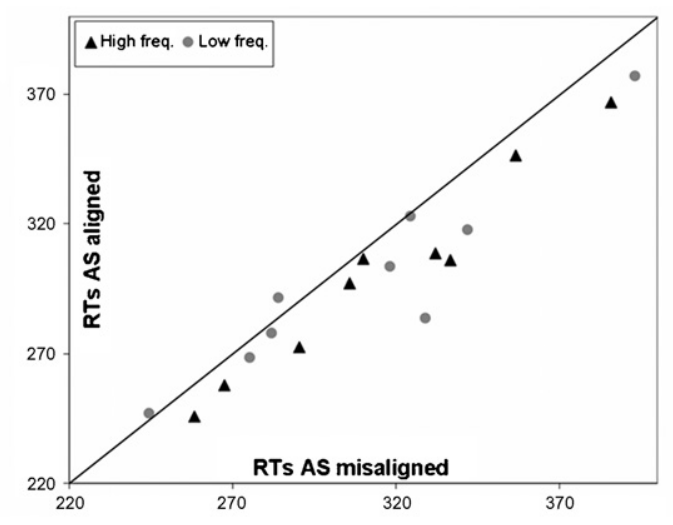

(C)

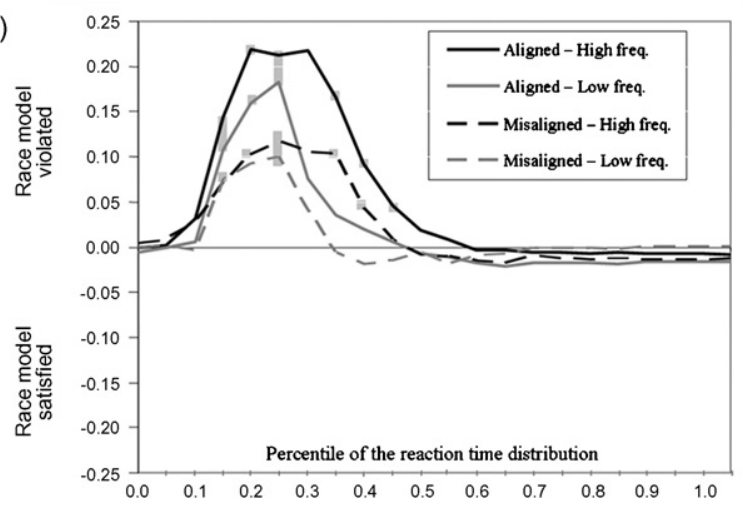

Fig. 4. Results of Experiment 3. (A) Mean RTs (in ms) for the conditions in which a low (left panel) or high frequency sound (right panel) was presented. The error bars indicate the standard errors of the mean. (B) Mean RTs (in milliseconds) for the AS aligned vs. AS misaligned conditions for each of the 15 participants. *marks a significant difference between conditions at $p<0.005$ level. (C) Results of applying Miller's (1982) inequality to the probability distributions of the RTs to the multisensory conditions.

enhanced for stimuli presented from the same versus opposite sides of a person's head, occur under at least certain conditions. The stimulated body surface, the relative distance to the auditory stimulus and the nature of the auditory stimuli seem to be relevant factors for eliciting this effect in the laboratory.

One of the critical findings to have emerged from Experiment 1 was that faster RTs were obtained when the stimuli were presented from the same side (aligned), as compared to when they were presented from different sides (misaligned), of the participant's head. This asymmetry between 'aligned' and 'misaligned' conditions was significant for the conditions in which the auditory stimulus was presented from close to the participant's head. In this case, while the 'aligned' condition clearly violated the race model, the violation for the 'misaligned' condition was close to fitting a 'separate-activation' model ${ }^{1}$. This spatial modulation of the RSE is

\footnotetext{
1 Though neural responses interactions may still be occurring (e.g. see Murray et al., 2001, for the case of unisensory visual pairs).
}

in line with those reported in other studies that have examined the RSE for audiovisual stimuli (e.g. Harrington \& Peck, 1998) and some studies of the RSE for visuotactile combinations (e.g. Diederich, Colonius, Bockhorst, \& Tabeling, 2003), although some authors have found no spatial modulation of visuotactile facilitation (e.g. Forster, Cavina-Pratesi, Aglioti, \& Berlucchi, 2002).

The results from Experiment 1 differ from previous studies of the RSE for auditory-somatosensory stimulus pairs (e.g. Murray et al., 2005; Zampini et al., 2007). The only evidence to date from psychophysical studies with healthy humans on the spatial modulation of responses to multisensory auditory-somatosensory stimuli was reported in a study using discrimination (TOJ) and distractor interference tasks (Kitagawa et al., 2005; see also Occelli Spence, \& Zampini, 2008, for a similar result in a TOJ task with blind participants). Therefore, the present results show for the first time an influence of spatial cues in auditory-somatosensory interactions when performing a simple detection task; a task that has been argued to be far less sensitive to attentional cuing effects than discrimination tasks (cf. Kitagawa \& Spence, 2006), arguing against the explanation that the spatial modulation of auditory-somatosensory interactions is simply dependent on the specific task requirements.

The spatial modulation of responses to multisensory auditory-somatosensory stimuli has been described previously in neuropsychological and neurophysiological studies (e.g. Farnè \& Làdavas, 2002; Graziano et al., 2004). In these studies, it was proposed that there are brain areas specialized for the integration of multisensory information emanating from events occurring in the immediate vicinity of the body and whose responses decrease as the distance between auditory (or visual) and tactile stimuli increases. It was also suggested (see Graziano et al., 2004) that different mechanisms for multisensory integration exist for the space near and far the body. According to this view, it is also possible to interpret the results obtained in Experiment 1 from a different perspective and suggest that there is a progressive slowing of RTs with increasing separation between the auditory and tactile stimuli, irrespective of the hemispace (same vs. different) from which they are presented. In Experiment 1, the spatial separation between the stimulated earlobe and the near loudspeaker situated in the opposite hemispace in the 'misaligned near' conditions was smaller than the separation between the stimulated earlobe and the far speaker at the same hemispace in the 'aligned far' conditions. The average RTs for the different combinations of stimuli were $302 \mathrm{~ms}$ (S.E. $=19$ ) for the aligned-near condition, $312 \mathrm{~ms}(\mathrm{~S} . \mathrm{E} .=21)$ for the aligned-far condition, $313 \mathrm{~ms}(\mathrm{~S} . \mathrm{E} .=21)$ for the misaligned-near condition and $317 \mathrm{~ms}($ S.E. $=22)$ for the misaligned-far condition. Thus, the paradigm utilized in this experiment defines three different distances: 'near' ('aligned near' conditions; with a distance between auditory (A) and somatosensory (S) stimuli of approximately $10 \mathrm{~cm}$ ), 'mid-range' ('aligned far' and 'misaligned near' conditions, with a distance between $A$ and $S$ stimuli of approximately 60 and $30 \mathrm{~cm}$, respectively) and 'far' ('misaligned far' condition, with a distance between $A$ and $S$ of approximately $80 \mathrm{~cm}$ ). A within-participant polynomial contrast with distance ('near', 'mid-range' or 'far') as the factor revealed a significant linear trend, $F_{(1,14)}=6.445, p=0.024$, thus showing that the spatial modulatory effect of auditory-somatosensory interactions was dependent on the spatial separation between somatosensory and auditory stimuli and not only on the hemifield were stimuli are positioned. Our results also indicate that the spatial modulation of auditory-somatosensory interactions is dependent on the particular body surface stimulated (in this case, the head) and not necessarily on the specific region of space where the stimuli are presented (i.e. the space around the head). In Experiment 1, where somatosensory stimuli were delivered to 
participants' earlobes, auditory-somatosensory integration was spatially modulated for auditory stimuli presented close to head. By contrast, in Experiment 2, no such spatial modulation was observed when the somatosensory stimuli were delivered to the participants' hands, even in the case where the participants' hands were placed by the participants' neck (i.e. in the space around the head).

Other studies have previously reported this dependence of auditory-somatosensory interactions on the particular body part stimulated (see Kitagawa \& Spence, 2006, for a review). For instance, Fu et al. (2003) examined body surface representations within area $\mathrm{CM}$ of macaques and showed that a high percentage of neurons were responsive to stimulation of the head and neck, whereas the percentage responsive to hand stimulation was minimal (cf. Fu et al., 2003; Fig. 2B). In a human neuroimaging study, Menning, Ackermann, Hertrich, and Mathiak (2005) examined the influence of tactile primes presented to both the face and hand on auditory responses. The results showed that spatial auditory attention was modulated by tactile priming only when tactile stimuli were presented from close to the face. Likewise, in a study of a patient suffering from alloesthesia, Ortigue et al. (2005) observed a mislocalization of touch for sounds presented close to the face, but not when the sounds were presented from close to the hand or foot. In addition, not only the part of the body stimulated, but also proprioceptive cues have been found to modulate the processing of spatially congruent auditory information (Simon-Dack \& Teder-Sälejärvi, 2008), although in the present study (Experiment 2) no significant effect of arm position (flexed vs. outstretched) was observed.

With regard to the results of Experiment 3, where auditory stimuli in different frequency ranges were used, our results suggest an asymmetry of the spatial multisensory modulation in favor of high frequency AS stimulus pairs. In this experiment, low frequency sounds were detected more rapidly in the auditory-alone conditions than high frequency sounds. However, this asymmetry in the RTs to high and low frequency sounds disappeared for the multisensory conditions. Moreover, the RSE for the multisensory conditions containing high-frequency sounds was modulated by stimuli spatial location, while the spatial modulatory effect was not significant for the stimuli pairs containing low-frequency sounds. Given that attenuation is greater for high- than for lowfrequency sounds, a high frequency sound may become a relatively weaker (i.e. less effective) stimulus. Given this fact, it could be argued that the greater multisensory gain observed for the highfrequency condition might simply reflect the principle of inverse effectiveness. In addition, this behavioral evidence might help to describe the nature and distribution of multisensory neurons. Our results suggest the possible existence of 'high frequency tuning' of the multisensory neurons responsible for auditory-somatosensory integration. Similarly, some anatomical and physiological evidence suggests there is a variation in the frequency tuning of the neurons in areas responsible for multisensory integration. For instance, animal research (e.g. with cats, Hirsch, Chan, \& Yin, 1985; Wise \& Irvine, 1983; with chinchillas, Mast \& Chung, 1973) has shown that the auditory RFs in the superior colliculus have a strong high frequency bias. A parallel line of argument is found in studies of the tonotopic organization of auditory cortices in non-human primates showing that posterior-most portions of core/primary auditory fields are likely to be tuned to higher frequency ranges (Kosaki, Hashikawa, He, \& Jones, 1997; Petkov, Kayser, Augath, \& Logothetis, 2006; Upadhyay et al., 2007) and are the predominant source of auditory input into the adjacent area CM where auditory-somatosensory convergence and interactions have been repeatedly documented (Hackett et al., 2007; Smiley et al., 2007).
Several other interpretations might explain the disparity observed between high and low frequency multisensory conditions. One possibility is that different brain areas or mechanisms are involved in auditory-somatosensory integration for auditory stimuli of different frequency bands, which in fact provide with different sound localization cues (interaural time differences for low frequency sound and interaural intensity differences for high frequency sounds; e.g. Blauert, 1997). There is evidence of different spatio-temporal brain dynamics involved in the processing and integration of different sound localization cues (Tardif, Murray, Meylan, Spierer, \& Clarke, 2006) and that might also extend to the case of multisensory integration. From an ecological perspective, it might be that the combination of the electrocutaneous stimulation with high frequency sounds is more significant in a natural environment, and therefore the spatial congruency of this multisensory event plays a major role in this case. In prior auditory research, it has been reported that below $60 \mathrm{~dB}$, low frequencies are more pleasant than are higher frequency sounds (Vitz, 1973) and that there is a correlation between the 'sharpness' of the sound (i.e. high frequency components) and emotional activation or arousal (see Västfjäll \& Kleiner, 2002, and references therein). Research on the emotional processing of sounds has shown that emotional responses to external stimulation are generated in many cases in early stages of stimulus processing, automatically and prior to awareness, and this enables the modulation of subsequent attentional and perception processes (for a review see Phelps \& LeDoux, 2005). Future research on auditory-somatosensory integration should address the influence of stimuli acoustic features and its ecological or emotional context.

Finally, in addition to the spatial alignment and proximity of stimulus pairs, task requirements might also influence multisensory integration (cf. Kitagawa \& Spence, 2006), such that the results when spatial information is task-relevant might diverge from those when spatial information is task-irrelevant (see also Spence \& McDonald, 2004). This possibility should be more directly examined in future research.

\section{Acknowledgments}

A.T. and A.V. received financial support from the PRESENCCIA project, an EU-funded Integrated Project under the IST programme (Project Number 27731). M.Z. was supported by a returning grant 'Rientro dei Cervelli' from the Ministero dell'Università e della Ricerca (Italy). M.M.M. receives financial support from the Swiss National Science Foundation (grant \#3100AO-118419). We thank to Dr. Hideyuki Ando for making the electrocutaneous stimulators.

\section{References}

Bergenheim, M., Johansson, H., Granlund, B., \& Pedersen, J. (1996). Experimental evidence for a sensory synchronization of sensory information to conscious experience. In S. R. Hameroff, A. W. Kaszniak, \& A. C. Scott (Eds.), Toward a science of consciousness: The first Tuscon discussions and debates (pp. 303-310). Cambridge, MA: MIT Press.

Blauert, J. (1997). Spatial hearing: The psychophysics of human sound localization. Cambridge, MA: MIT Press.

Calvert, G. A., Spence, C., \& Stein, B. E. (2004). The handbook of multisensory processes. Cambridge, MA: MIT Press.

Diederich, A., Colonius, H., Bockhorst, D., \& Tabeling, S. (2003). Visual-tactile spatial interaction in saccade generation. Experimental Brain Research, 148, 328-337.

Driver, J., \& Noesselt, T. (2008). Multisensory interplay reveals crossmodal influences on 'sensory-specific' brain regions, neural responses, and judgments. Neuron, 57, $11-23$.

Farnè, A., \& Làdavas, E. (2002). Auditory peripersonal space in humans. Journal of Cognitive Neuroscience, 14, 1030-1043.

Forster, B., Cavina-Pratesi, C., Aglioti, S. M., \& Berlucchi, G. (2002). Redundant target effect and intersensory facilitation from visual-tactile interactions in simple reaction time. Experimental Brain Research, 143, 480-487. 
Fu, K. M., Johnston, T. A., Shah, A. S., Arnold, L., Smiley, J., Hackett, T. A., Garraghty, P. E., \& Schroeder, C. E. (2003). Auditory cortical neurons respond to somatosensory stimulation. Journal of Neuroscience, 23, 7510-7515.

Ghazanfar, A. A., \& Schroeder, C. E. (2006). Is neocortex essentially multisensory? Trends in Cognitive Sciences, 10, 278-285.

Graziano, M. S. A., Gross, C. G., Taylor, C. S. R., \& Moore, T. (2004). A system of multimodal areas in the primate brain. In C. Spence \& J. Driver (Eds.), Multisensory space and multisensory attention (pp. 51-67). Oxford: Oxford University Press.

Graziano, M. S. A., Reiss, L. A. J., \& Gross, C. G. (1999). A neuronal representation of the location of nearby sounds. Nature, 397, 428-430.

Hackett, T. A., Smiley, J. F., de la Mothe, L. A., Ulbert, I., Karmos, G., \& Schroeder, C. E. (2007). Connections of the caudal STP in macaques. Journal of Comparative Neurology, 502, 924-952.

Harrington, L. K., \& Peck, C. K. (1998). Spatial disparity affects visual-auditory interactions in human sensorimotor processing. Experimental Brain Research, 122 247-252.

Hirsch, J. A., Chan, J. C. K., \& Yin, T. C. T. (1985). Responses of neurons in the cat's superior colliculus to acoustic stimuli. I. Monaural and binaural response properties. Journal of Neurophysiology, 53, 726-745.

Kayser, C., Petkov, C. I., Augath, M., \& Logothetis, N. (2005). Integration of touch and sound in auditory cortex. Neuron, $48,373-384$.

Kitagawa, N., \& Spence, C. (2006). Audiotactile multisensory interactions in information processing. Japanese Psychological Research, 48, 158-173.

Kitagawa, N., Zampini, M., \& Spence, C. (2005). Audiotactile interactions in near and far space. Experimental Brain Research, 166, 528-537.

Kosaki, H., Hashikawa, T., He, J., \& Jones, E. G. (1997). Tonotopic organization of auditory cortical fields delineated by parvalbumin immunoreactivity in macaque monkeys. Journal of Comparative Neurology, 386, 304-316.

Làdavas, E., \& Farnè, A. (2004). Neuropsychological evidence for multimodal representations of space near specific body parts. In C. Spence \& J. Driver (Eds.) Multisensory space and multisensory attention (pp. 69-98). Oxford: Oxford University Press.

Lakatos, P., Chen, C. M., O'Connell, M. N., Mills, A., \& Schroeder, C. E. (2007). Neuronal oscillations and multisensory interaction in primary auditory cortex. Neuron, 53 , 279-292.

Lamarre, Y., Busby, L., \& Spidalieri, G. (1983). Fast ballistic arm movements triggered by visual, auditory, and somesthetic stimuli in the monkey. I. Activity of precentral cortical neurons. Journal of Neurophysiology, 50, 1343-1358.

Martuzzi, R., Murray, M. M., Michel, C. M., Thiran, J. P., Maeder, P. P., Clarke, S., \& Meuli, R. A. (2007). Multisensory interactions within human primary cortices revealed by BOLD dynamics. Cerebral Cortex, 17, 1672-1679.

Mast, T. E., \& Chung, D. Y. (1973). Binaural interaction in the superior colliculus of the chinchilla. Brain Research, 62, 227-230.

Menning, H., Ackermann, H., Hertrich, I., \& Mathiak, K. (2005). Spatial auditory attention is modulated by tactile priming. Experimental Brain Research, 164, 41-47.

Miller, J. (1982). Discrete versus continuous stage models of human information processing: In search of partial output. Journal of Experimental Psychology: Human Perception \& Performance, 8, 273-296.

Murray, M. M., Foxe, J. J., Higgins, B. A., Javitt, D. C., \& Schroeder, C. E. (2001). Visuospatial neural response interactions in early visual cortical processing during a simple reaction time task: A high-density electrical mapping study. Neuropsychologia, 39, 828-844.

Murray, M. M., Molholm, S., Michel, C. M., Heslenfeld, D. J., Ritter, W., Javitt, D. C., Schroeder, C. E., \& Foxe, J. J. (2005). Grabbing your ear: Auditory-somatosensory multisensory interactions in early sensory cortices are not constrained by stimulus alignment. Cerebral Cortex, 15, 963-974

Occelli, V., Spence, C., \& Zampini, M. (2008). Audiotactile temporal order judgments in sighted and blind individuals. Neuropsychologia, doi:10.1016/j. neuropsychologia.2008.05.023

Ortigue, S., Jabaudon, D., Landis, T., Michel, C. M., Maravita, A., \& Blanke, O. (2005). Preattentive interference between touch and audition: A case study on multisensory alloesthesia. Neuroreport, 16, 865-868.

Petkov, C. I., Kayser, C., Augath, M., \& Logothetis, N. K. (2006). Functional imaging reveals numerous fields in the monkey auditory cortex. PLoS Biol, 4(7), e215.

Phelps, E. A., \& LeDoux, J. E. (2005). Contributions of the amygdala to emotion processing: From animal models to human behavior. Neuron, 48, 175-187.

Raab, D. (1962). Statistical facilitation of simple reaction times. Transactions of the New York Academy of Sciences, 24, 574-590.

Romei, V., Murray, M. M., Merabet, L. B., \& Thut, G. (2007). Occipital transcranial magnetic stimulation has opposing effects on visual and auditory stimulus detection: Implications for multisensory interactions. Journal of Neuroscience, 27, 11465-11472.

Simon-Dack, S. L., \& Teder-Sälejärvi, W. A. (2008). Proprioceptive cues modulate further processing of spatially congruent auditory information. A high-density EEG study. Brain Research, 1220, 171-178.

Smiley, J. F., Hackett, T. A., Ulbert, I., Karmos, G., Javitt, D. C., \& Schroeder, C. E. (2007) Multisensory convergence in auditory cortex. I. Cortical connections of the caudal superior temporal plane. Journal of Comparative Neurology, 502, 894-923.

Spence, C. (2007). Audiovisual multisensory integration. Acoustical Science and Technology, 28, 61-70.

Spence, C., \& Driver, J. (Eds.). (2004). Multisensory space and multisensory attention. Oxford: Oxford University Press.

Spence, C., \& McDonald, J. (2004). The crossmodal consequences of the exogenous spatial orienting of attention. In G. A. Calvert, C. Spence, \& B. E. Stein (Eds.), The handbook of multisensory processing (pp. 3-25). Cambridge, MA: MIT Press.

Stein, B. E., \& Meredith, M. A. (1993). The merging of the senses. Cambridge, MA: MIT Press.

Tardif, E., Murray, M. M., Meylan, R., Spierer, L., \& Clarke, S. (2006). The spatiotemporal brain dynamics of processing and integrating sound localization cues in humans. Brain Research, 1092, 161-176.

Upadhyay, J., Ducros, M., Knaus, T. A., Lindgren, K. A., Silver, A., Tager-Flusberg, H., \& Kim, D. S. (2007). Function and connectivity in human primary auditory cortex: A combined fMRI and DTI study at 3 Tesla. Cerebral Cortex, 17, 2420-2432.

Västfjäll, D., \& Kleiner, M. (2002). Emotion in product sound design. In Proceedings of Journées Design Sonore.

Vitz, P. C. (1973). Preference for tones as a function of frequency and intensity. Perception \& Psychophysics, 11, 84-88.

Wallace, M. T., \& Stein, B. E. (2007). Early experience determines how the senses will interact. Journal of Neurophysiology, 97, 921-926.

Welch, R. B., \& Warren, D. H. (1986). Intersensory interactions. In K. R. Boff, L. Kaufman, \& J. P. Thomas (Eds.), Handbook of perception and performance Sensory processes and perception. New York: Wiley, pp. 25-31, 25-36.

Wise, L. Z., \& Irvine, D. R. F. (1983). Auditory response properties of neurons in deep layers of cat superior colliculus. Journal of Neurophysiology, 49, 674-685.

Zampini, M., Brown, T., Shore, D. I., Maravita, A., Röder, B., \& Spence, C. (2005). Audiotactile temporal order judgments. Acta Psychologica, 118, 277-291.

Zampini, M., Torresan, D., Spence, C., \& Murray, M. M. (2007). Audiotactile multisensory interactions in front and rear space. Neuropsychologia, 45, 1869-1877. 